
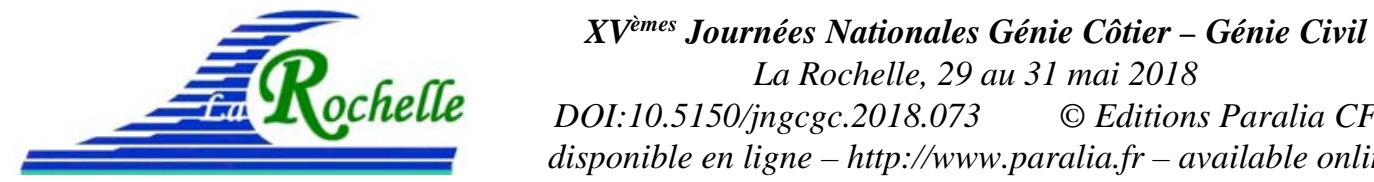

DOI:10.5150/jngcgc.2018.073 (C) Editions Paralia CFL

disponible en ligne - http://www.paralia.fr - available online

\title{
Coordination de la recherche et des études inter-estuaires : comprendre les estuaires pour mieux les préserver.
}

\author{
Valérie FOUSSARD ${ }^{1}$, Maïa AKOPIAN ${ }^{2}$
}

1. FR CNRS 3730 SCALE, Université de Rouen Normandie, UMR 6143 CNRS - M2C, Place Emile Blondel, 76821 Mont-Saint-Aignan, France.

valerie.foussard@univ-rouen.fr

2. Agence française pour la biodiversité, DREC, DRDI,

5, square Félix Nadar, 94300 Vincennes, France.

maia.akopian@afbiodiversite.fr

\section{Résumé :}

L'Agence Française pour la Biodiversité (AFB) soutient des projets scientifiques répondant à des besoins opérationnels (appui aux politiques publiques et à la gestion des milieux aquatiques, développement de la connaissance). La "Coordination de la recherche et des études inter-estuaires" mise en place par l'AFB et ses partenaires en 2012, a pour ambition de : favoriser les échanges entre acteurs des estuaires, développer la connaissance, venir en appui aux partenaires en développant des outils opérationnels, et valoriser les travaux scientifiques et techniques réalisés sur les estuaires.

Depuis plusieurs années, la mise en œuvre de la Directive Cadre sur l'Eau (DCE) constitue un des enjeux prioritaires au niveau national. Pour y répondre, plusieurs projets sont menés afin de définir des méthodes d'évaluation et de surveillance de l'état écologique des estuaires, comprendre l'évolution morphologique des estuaires sous l'effet des facteurs naturels et anthropiques, et dégager des pistes de restaurations écologiques. Ces collaborations scientifiques ont notamment permis de progresser sur :

a) Les indicateurs de qualité DCE et les stratégies de suivi pour : l'oxygène dissous, les invertébrés benthiques, l'hydromorphologie au regard de la biologie et au travers des pressions anthropiques ;

b) Une meilleure prise en compte du réseau trophique et du fonctionnement estuarien : évaluation de la production primaire (via le microphytobenthos), réflexion sur des propositions de mesures de gestion tenant compte du fonctionnement estuarien et des interactions trophiques ;

c) L'acquisition automatisée de données à large échelle (télédétection, imagerie acoustique) de la flore (microphytobenthos, schorre) ou de la nature des sédiments ;

d) L'analyse des retours d'expérience de restauration écologique estuarienne par une analyse critique de ces actions, des objectifs poursuivis et des outils employés.

\section{Mots-clés :}

Estuaires, Recherche appliquée, Evaluation écologique, Acquisition de connaissances. 


\section{Thème 6 - Gestion durable des zones littorales et estuariennes}

\section{Introduction}

Zones de transition entre les eaux douces et marines, les estuaires sont des écosystèmes complexes possédant des caractéristiques hydrologiques, morphologiques et sédimentaires très variables dans le temps et l'espace. Bien qu'ils présentent d'importantes contraintes environnementales naturelles pour les organismes vivants (gradients de salinité, turbidité, variations des hauteurs d'eau...), les estuaires sont des milieux très productifs. Ils jouent un rôle essentiel dans le cycle de vie de nombreuses espèces benthiques, démersales, pélagiques et/ou migratrices.

Les estuaires sont également des territoires très convoités par l'Homme pour développer ses activités (navigation, pêche, urbanisation...). Ces usages sont à l'origine de pressions anthropiques susceptibles de perturber à grande échelle, le fonctionnement écologique des estuaires, qu'elles soient directes (e.g. endiguements, dragages) ou diffuses (e.g. rejets de substances chimiques, amplification des apports en matières en suspension par érosion des sols nus).

Afin d'appréhender le fonctionnement des estuaires, évaluer l'impact des pressions anthropiques sur ces systèmes et développer des outils de gestion adaptés, l'Agence Française pour la Biodiversité (AFB) a mis en place la "Coordination de la recherche et des études inter-estuaires" avec l'appui de divers partenaires. Cette Coordination interestuaires a pour ambition de (1) rassembler les acteurs majeurs des estuaires de la façade Manche/Atlantique (Agences de l'eau, structures d'interface sciences/gestion, organismes de recherche) autour de problématiques communes, (2) développer et valoriser la connaissance sur ces milieux complexes et (3) construire des outils opérationnels répondant prioritairement aux politiques publiques (e.g. Directive Cadre sur l'Eau). Depuis 2013, l'animation de cette coordination est réalisée dans le cadre d'un partenariat entre l'AFB et la Fédération de Recherche "Sciences Appliquées à L'Environnement" (FR CRNS 3730 SCALE) à l'Université de Rouen Normandie.

\section{Contexte et objectif de la Coordination inter-estuaires}

L'AFB soutient les projets scientifiques répondant à des besoins opérationnels relatifs aux milieux aquatiques (appui aux politiques publiques et à la gestion des milieux aquatiques, développement de la connaissance). Ces dernières années, la mise en œuvre des directives européennes et tout particulièrement la Directive Cadre sur l'Eau (DCE) constitue un des enjeux prioritaires au niveau national. Ainsi, divers projets sont menés dans le cadre de la Coordination inter-estuaires pour venir en appui aux Agences de l'eau (Artois-Picardie, Seine-Normandie, Loire-Bretagne et Adour-Garonne) chargées de la mise en œuvre de la DCE sur leur territoire, étape par étape (Figure 1).

Les travaux en cours concernent principalement :

a) L'évaluation de l'état des masses d'eau de transition : développement d'indicateurs de qualité DCE-compatible et leur intercalibration européenne ; 


\section{XVèmes Journées Nationales Génie Côtier - Génie Civil \\ La Rochelle, 29 au 31 mai 2018}

b) La surveillance des milieux : détermination de stratégies de suivi pertinentes vis-àvis des indicateurs développées en parallèle et des spécificités des estuaires ;

c) Revue systématique des restaurations écologiques: aide aux gestionnaires pour définir des programmes de mesures visant l'amélioration de la qualité des milieux ;

d) La comparaison des résultats obtenus par divers indicateurs (DCE, de biodiversité et trophiques) pour une meilleure appréhension de ce qu'ils traduisent et aider à la définition de mesures de gestion adaptées.

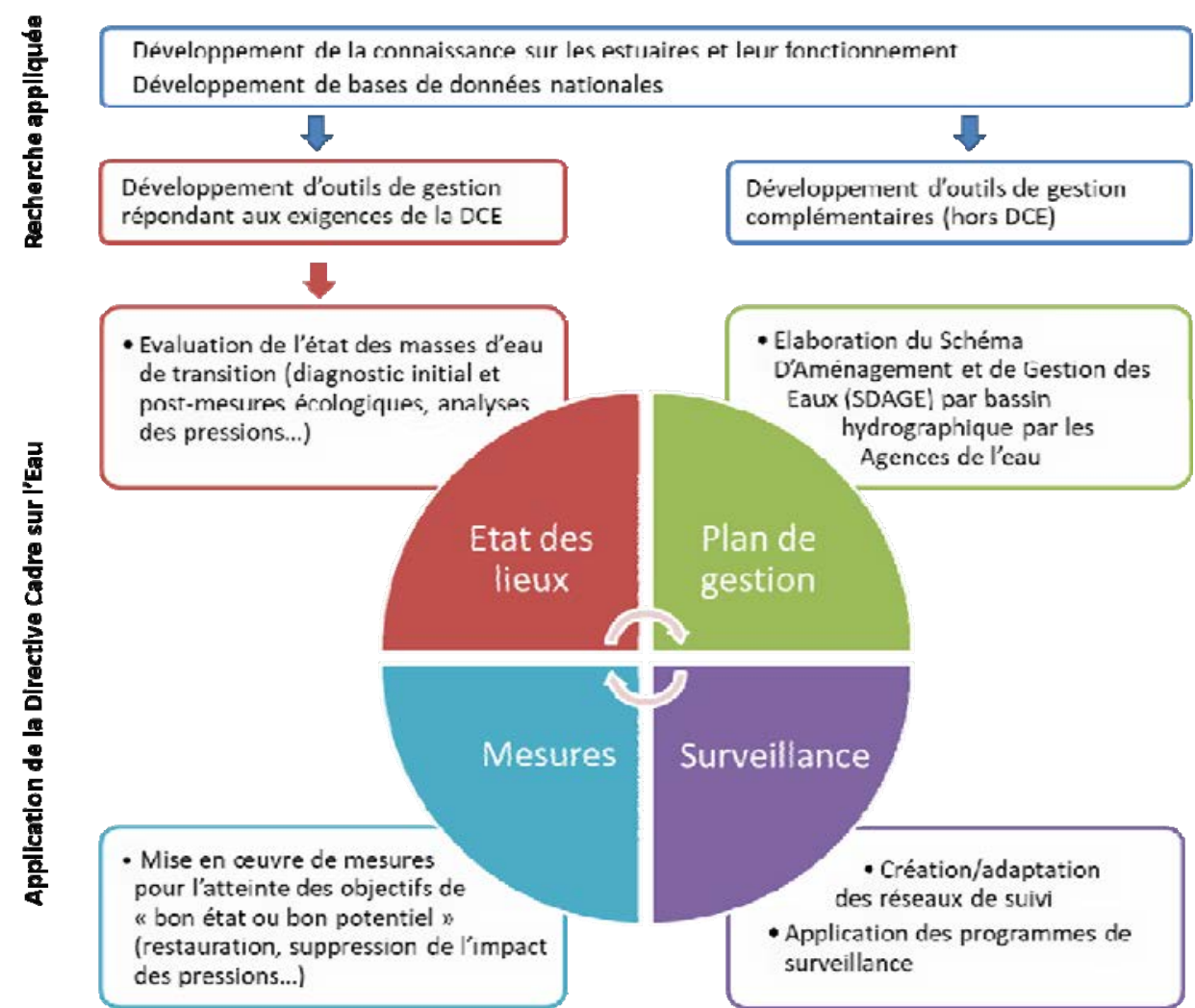

Figure 1. Etapes simplifiées de mise en œuvre de la DCE et lien avec les travaux menés par la Coordination inter-estuaires.

\section{Activités scientifiques et techniques de la Coordination inter-estuaires}

La définition de méthodes d'évaluation de la qualité écologique des estuaires et la mise en place d'une surveillance pertinente des milieux sont des étapes primordiales dans la mise en œuvre de la DCE. Compte tenu des spécificités des estuaires (gradients spatiotemporels des conditions), les indicateurs développés pour les milieux adjacents (fluviaux ou côtiers) ne peuvent être transposés directement aux milieux estuariens. Par conséquent, l'AFB par le biais de la Coordination inter-estuaires, a mobilisé des moyens importants pour développer de nouvelles méthodes lorsque ces outils ne pouvaient être adaptés aux estuaires (projets listés dans le tableau 1). 


\section{Thème 6 - Gestion durable des zones littorales et estuariennes}

Tableau 1. Projets en collaboration avec l'AFB via la Coordination inter-estuaires Les pastilles colorées font référence aux étapes de la figure 1.

\begin{tabular}{|c|c|c|c|c|}
\hline \multicolumn{2}{|c|}{ Thématique/approche } & \multirow{2}{*}{$\begin{array}{l}\text { Projet en inter-estuaires } \\
\text { Test d'applicabilité des indicateurs } \\
\text { européens sur les estuaires français et } \\
\text { intercalibration }\end{array}$} & \multirow{2}{*}{$\begin{array}{l}\text { Equipe(s) impliquée(s) } \\
\text { UMR } 5805 \text { EPOC - Univ. } \\
\text { Bordeaux / IRSTEA }\end{array}$} & \multirow{2}{*}{$\begin{array}{l}\text { Réalisation } \\
2017 / 2018\end{array}$} \\
\hline $\begin{array}{l}\text { Evaluation } \\
\text { biologique } \\
\text { (DCE) }\end{array}$ & $\begin{array}{l}\text { Invertébrés } \\
\text { benthiques }\end{array}$ & & & \\
\hline & $\begin{array}{l}\text { Microphyto- } \\
\text { benthos }\end{array}$ & $\begin{array}{l}\text { Evaluation de la pertinence du } \\
\text { microphytobenthos comme indicateur } \\
\text { biologique en estuaires }\end{array}$ & $\begin{array}{l}\text { EA } 2160 \text { MMS - Univ. de } \\
\text { Nantes }\end{array}$ & $2015 / 2019$ \\
\hline \multirow{2}{*}{$\begin{array}{l}\text { Evaluation } \\
\text { physico- } \\
\text { chimique } \\
\text { (DCE) }\end{array}$} & $\begin{array}{l}\text { Oxygène } \\
\text { dissous }\end{array}$ & $\begin{array}{l}\text { Caractérisation des zones hypoxiques des } \\
\text { estuaires de la Loire et de la Gironde en } \\
\text { vue de développer un indicateur DCE }\end{array}$ & $\begin{array}{l}\text { IRSTEA / UMR CNRS } \\
\text { EPOC, Univ. Bordeaux/ } \\
\text { GIP Loire Estuaire }\end{array}$ & $\begin{array}{l}2014- \\
2017 / 2018\end{array}$ \\
\hline & $\begin{array}{l}\text { Oxygène } \\
\text { dissous }\end{array}$ & $\begin{array}{l}\text { Seuil de tolérance de la faune estuarienne } \\
\text { à la concentration en oxygène dissous }\end{array}$ & $\begin{array}{l}\text { UMR SEBIO - Univ. Le } \\
\text { Havre Normandie }\end{array}$ & 2018 \\
\hline \multirow[t]{3}{*}{$\begin{array}{l}\text { Evaluation } \\
\text { hydromorpho- } \\
\text { logique (DCE) }\end{array}$} & \multirow{2}{*}{$\begin{array}{l}\text { Pressions } \\
\text { anthropiques }\end{array}$} & $\begin{array}{l}\text { Evaluation des estuaires en très bon état } \\
\text { hydromorphologique au regard des } \\
\text { pressions subies }\end{array}$ & BRGM & $2016 / 2018$ \\
\hline & & $\begin{array}{l}\text { Caractérisation des masses d'eau } \\
\text { fortement modifiées en estuaires (DCE) }\end{array}$ & BRGM & 2018 \\
\hline & $\begin{array}{l}\text { Morphologie, } \\
\text { salinité }\end{array}$ & $\begin{array}{l}\text { Test d'indicateurs Hydro-Morpho- } \\
\text { Sédimentaires pour évaluer l'état HMS } \\
\text { des estuaires }\end{array}$ & $\begin{array}{l}\text { UMR CNRS } 5805 \text { EPOC / } \\
\text { IFREMER / FR SCALE } \\
\text { (Coord. inter-estuaires) }\end{array}$ & $\begin{array}{l}2013 / 2015 \\
\text { Stage } 2018\end{array}$ \\
\hline $\begin{array}{l}\text { Etude } \\
\text { comparative }\end{array}$ & Biologie & $\begin{array}{l}\text { Analyse d'indicateurs de biodiversité, } \\
\text { d'état écologique et de fonctionnement } \\
\text { trophique des estuaires et propositions de } \\
\text { mesures de gestion }\end{array}$ & IRSTEA & $2016 / 2018$ \\
\hline $\begin{array}{l}\text { Caractérisation } \\
\text { habitat }\end{array}$ & $\begin{array}{l}\text { Nature des } \\
\text { sédiments }\end{array}$ & $\begin{array}{l}\text { Faisabilité de caractériser la nature des } \\
\text { sédiments estuariens par des méthodes } \\
\text { automatiques, }\end{array}$ & $\begin{array}{l}\text { UMR CNRS } 6143 \text { M2C - } \\
\text { Université Rouen/Caen } \\
\text { Normandie }\end{array}$ & $2018 / 2020$ \\
\hline $\begin{array}{l}\text { Restauration } \\
\text { écologique }\end{array}$ & $\begin{array}{l}\text { Retours } \\
\text { d'expérience }\end{array}$ & $\begin{array}{l}\text { Analyse des outils employés dans les } \\
\text { restaurations (définition des objectifs, } \\
\text { état des lieux, évaluation) }\end{array}$ & $\begin{array}{l}\text { BRGM / GIP Seine-Aval / } \\
\text { FR SCALE (Coord. inter- } \\
\text { estuaires) }\end{array}$ & $2015 / 2017$ \\
\hline
\end{tabular}

Les rapports intermédiaires ou finaux ainsi que les bases de données produites au cours de ces projets sont accessibles sur le site de l'AFB (http://www.onema.fr/comprendreles-milieux-estuariens-pour-mieux-les-preserver). 


\section{XVèmes Journées Nationales Génie Côtier - Génie Civil \\ La Rochelle, 29 au 31 mai 2018}

\section{Qualification des habitats estuariens : exemple d'application de l'indicateur relatif à la proportion et continuité des grands types d'habitats}

\subsection{Proportion de zones intertidales dans les masses d'eau de transition}

Les conditions hydro-morpho-sédimentaires (HMS) des estuaires liées aux influences de la marée et du fleuve, créent des habitats particuliers d'un grand intérêt écologique. En l'occurrence, les zones intertidales quel que soit leur type (vasière, banc de sable, marais intertidal...), assurent un grand nombre de fonctions écologiques relatives au cycle de vie des espèces (e.g. alimentation, nourricerie) ou encore aux flux de matières et d'énergie (e.g. dégradation ou stockage des nutriments ou de matières en suspension). Selon la typologie des estuaires (morphologie structurale, régimes de marée et fluvial) et les pressions HMS qu'ils peuvent subir (e.g. cloisonnement par endiguement), la proportion de zones intertidales par rapport à la surface totale de l'estuaire peut être très importante (figure 2) permettant ainsi d'optimiser les possibilités d'accomplissement des fonctions écologiques associées à ce type d'habitat. Cela étant, la réalisation de ces fonctions ne dépend pas uniquement de la surface des habitats. D'autres critères entrent en jeu tels que les caractéristiques physiques et trophiques de ces habitats (e.g. nature du substrat, ressources trophiques disponibles) ou encore la continuité et l'accessibilité de ces habitats.

L'impact des pressions anthropiques sur le fonctionnement des estuaires est également à considérer; c'est pourquoi les évaluations HMS des MET et plus largement leurs évaluations écologiques sont réalisées au regard des pressions exercées.

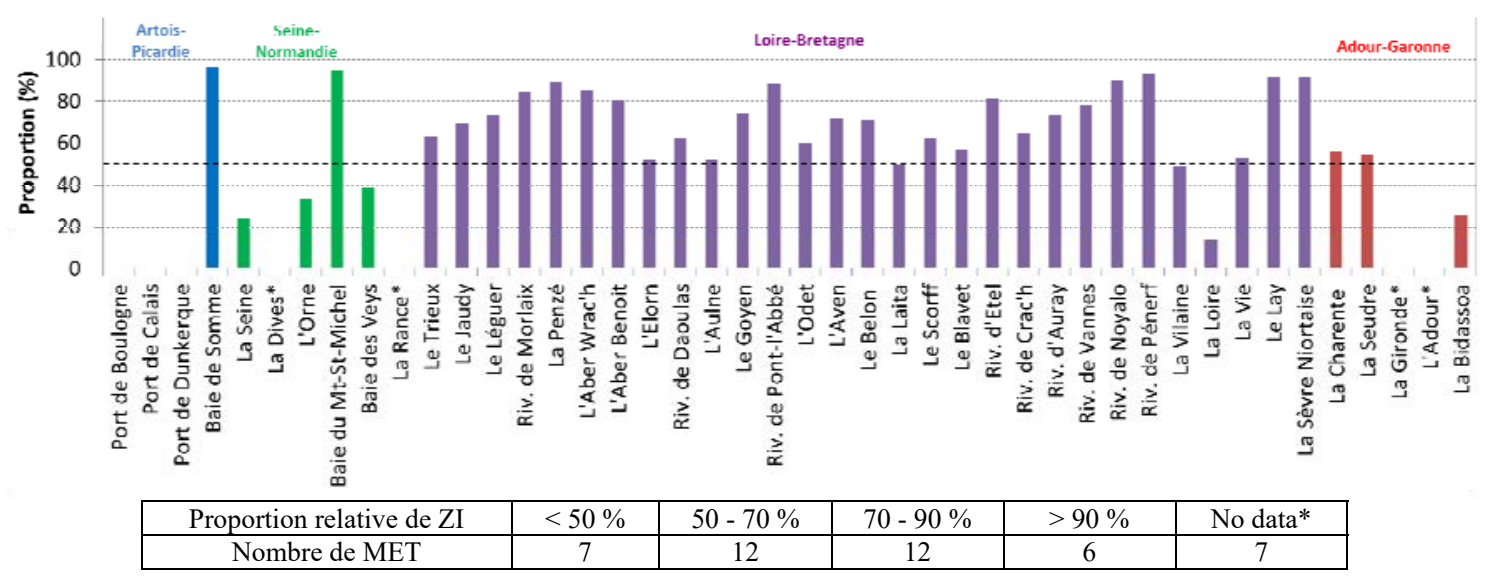

Figure 2. Proportion de zones intertidales (ZI) relative à la surface totale des masses d'eau de transition (MET) (SOTTOLICHIO et al., 2014)

\subsection{Continuité longitudinale des zones intertidales}

Dans l'optique d'évaluer la continuité amont/aval des habitats intertidaux et subtidaux, un indicateur appelé "indicateur hypsométrique" a été développé dans le cadre d'un 


\section{Thème 6 - Gestion durable des zones littorales et estuariennes}

groupe de travail HMS coordonné par l'Ifremer et l'UMR EPOC à l'Université de Bordeaux. Il consiste à déterminer la proportion de zones intertidales et subtidales, le long de chaque estuaire (SOTTOLICHIO et al., 2014 ; LE HIR \& BOUVIER, 2015 ; BOUVIER et al., 2016). Cet outil d'évaluation est en cours d'application par la Coordination inter-estuaires (FR SCALE) sur l'ensemble des milieux de transition de la façade Manche/Atlantique. Cet exercice est réalisé à partir des surfaces d'habitats obtenues par digitalisation des orthophotographies littoral V2 mises à disposition par le Ministère chargé de l'environnement. La figure 3 présente deux exemples d'application de l'indicateur hypsométrique sur l'estuaire de la Laïta (Bretagne, Côte d'Armor) et l'estuaire du Lay (Pays-de-la-Loire, Vendée).

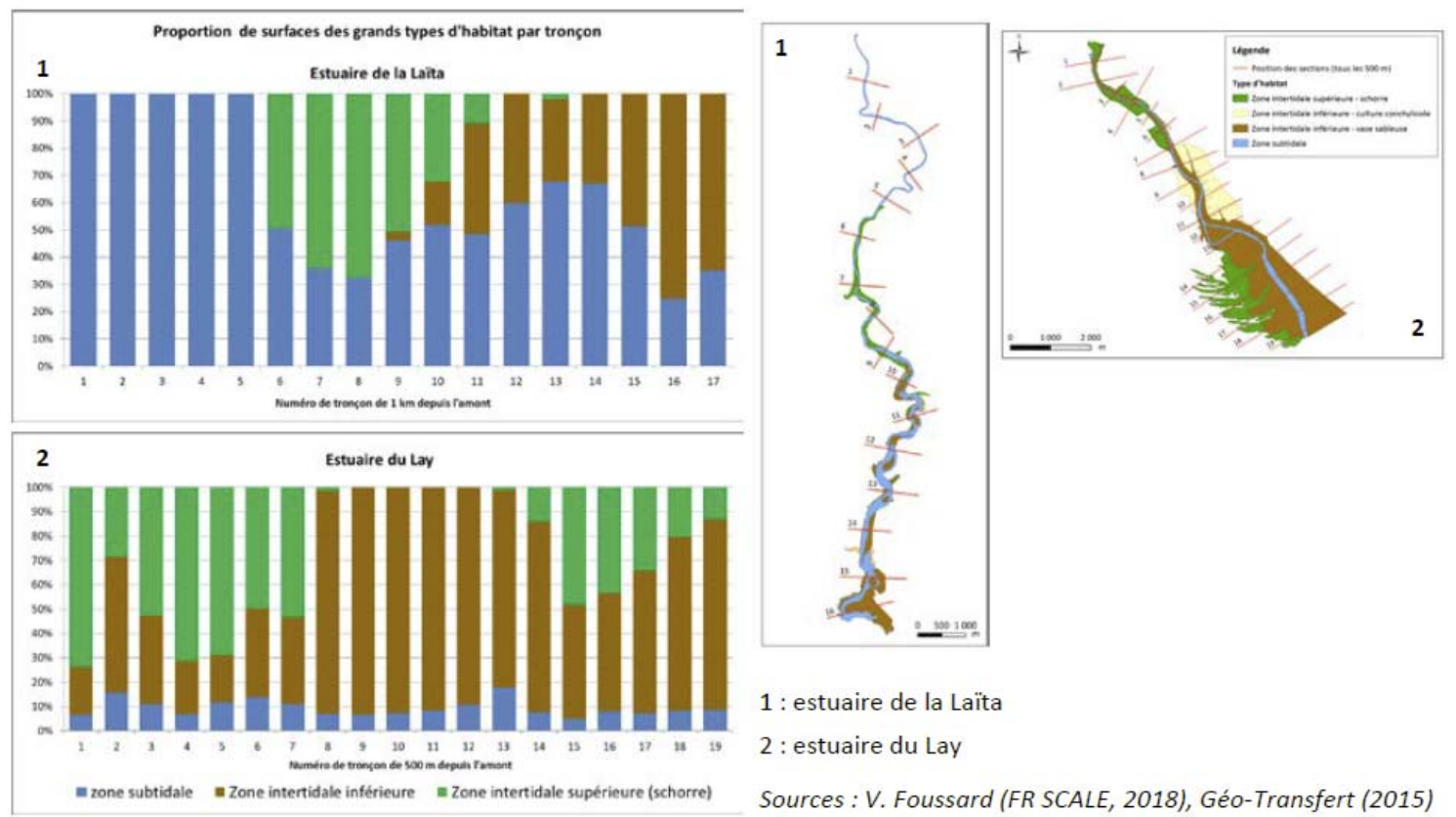

Figure 3. Proportion amont/aval des grands types d'habitats et sectorisation des estuaires de la Laïta et du Lay (SOTTOLICHIO et al., 2014).

Ces deux estuaires présentent des morphologies différentes :

a) Estuaire de la Laïta : la partie amont est constituée essentiellement de zones subtidales ("couloir" immergé). Les zones intertidales inférieures (composées de sables, vases ou mélange sablo-vaseux) où se concentrent les ressources trophiques, sont localisées à l'aval de l'estuaire et représentent $50 \%$ de la surface totale.

b) Estuaire du Lay: les zones intertidales inférieures (composées majoritairement de vases sableuses) et supérieures (schorre) représentent un peu de plus de $90 \%$ de la surface totale et sont réparties le long de l'estuaire. Le secteur situé entre les tronçons 7 et 12 est principalement occupé par de la conchyliculture.

A terme, l'objectif serait de comparer les étendues, la continuité des grands types d'habitat et leur accessibilité selon le cycle de marée afin de déterminer si leur usage par 


\section{XVèmes Journées Nationales Génie Côtier - Génie Civil \\ La Rochelle, 29 au 31 mai 2018}

les organismes est optimal (e.g. positon des zones d'alimentation optimales compte tenu des modes de vie des poissons). Pour cela, ces informations doivent être croisées avec diverses données biologiques et environnementales afin d'une part, mieux appréhender l'utilisation des habitats par les organismes au cours de leur cycle de vie et d'autre part, mieux évaluer la qualité écologique des estuaires au sens large (physique, biologique et physico-chimique).

L'influence des pressions anthropiques est également à considérer bien que leurs impacts sur les peuplements et leur habitat soient difficiles à différencier des effets des facteurs naturels. Ceci est notamment lié au caractère ubiquiste des espèces rencontrées en estuaires ("Estuarine Quality paradox").

\section{Conclusion}

Le fonctionnement des estuaires est complexe à appréhender en raison de l'importante variabilité spatio-temporelle des conditions environnementales engendrée par la rencontre des eaux fluviales et marines. Les organismes vivant dans les estuaires sont généralement ubiquistes et sont par nature résistants à ces contraintes naturelles fortes mais également aux impacts des pressions anthropiques exercées sur ces écosystèmes. La distinction entre les effets des facteurs naturels et les impacts des pressions anthropiques locales ou liées au changement climatique, est par conséquent, difficile à faire, rendant tout aussi complexe la définition de méthodes d'évaluation reflétant au mieux la qualité des estuaires.

Malgré ce constat, depuis sa création, la Coordination inter-estuaires a permis de progresser sur diverses problématiques nationales au travers des projets en cours visant:

c) le développement d'indicateurs de qualité DCE (sur l'oxygène dissous, les invertébrés benthiques, l'hydromorphologie);

d) Une meilleure prise en compte du réseau trophique et du fonctionnement estuarien dans les évaluations et les mesures de gestion;

e) Le développement de méthode de surveillance automatisée à large échelle (télédétection, imagerie acoustique) pour le microphytobenthos, le schorre et la nature des sédiments ;

f) La mise en commun des enseignements tirés des actions de restauration écologique en estuaires menées à l'échelle internationale.

\section{Références bibliographiques}

BOUVIER C., SOTTOLICHIO A., HANQUIEZ V., LE HIR P., FOUSSARD V. (2016). Développement d'indicateurs hydro-morphologiques pour l'application de la Directive Cadre Eau dans les eaux de transition estuariennes - Phase 2 : Tests des indicateurs morphologiques sur les Grands estuaires Français. Rapport intermédiaire Université de Bordeaux - Onema. Disponible en ligne: http://www.onema.fr/evaluation-de-la-qualiteecologique-des-estuaires 
Thème 6 - Gestion durable des zones littorales et estuariennes

LE HIR P., BOUVIER C. (2015). Développements d'indicateurs hydro-morphosédimentaires pour l'application de la Directive Cadre Eau dans les eaux de transition estuariennes - Phase 2 : Tests des indicateurs sur des configurations d'estuaires schématiques à l'aide d'une modélisation 3D. Rapport intermédiaire Ifremer - Onema Disponible en ligne : http://www.onema.fr/evaluation-de-la-qualite-ecologique-des-estuaires

SOTTOLICHIO A., FOUSSARD V., KERVELlA S., CURTI C., ARRIAGADA J., HANQUIEZ V. (2014). Développement d'indicateurs HydroMorphoSédimentaires pour les estuaires - Inventaire des caractéristiques des estuaires. Rapport intermédiaire Université de Bordeaux - Onema. Disponible en ligne: http://www.onema.fr/evaluation-de-la-qualiteecologique-des-estuaires 